\title{
Timing Recovery for Ultra Wideband Systems
}

\author{
Mohammad Ghavami Reza S. Dilmaghani \\ King's College London \\ Ultra Wideband Communications Group \\ Strand, London WC2R 2LS, UK \\ Tel: +44 (0)2078482596 \\ Fax: +44 (0)207 8482664
}

\begin{abstract}
This paper examines the problems of symbol timing estimation and timing recovery for ultra wideband impulse radio signals. Two different approaches based on the maximum likelihood technique are investigated. The first approach is based on derivative matched filter and the second one takes advantage of the early-late technique. Furthermore, two non-coherent timing recovery circuits are proposed and discussed. The first circuit is a digital delay-locked loop (DDLL) and the second circuit is based on early-late technique. Finally, performance analysis of the proposed techniques for IEEE 802.15.3a channel models in terms of the minimum squared error (MSE) is provided.
\end{abstract}

\section{INTRODUCTION}

Ultra wideband (UWB) communication systems, which are based on impulse radio, employ short duration pulses with fractional bandwidth of greater than 20\% [1]. Transmission of information based on UWB signals is considered a good candidate for short range, high data rate applications such as wireless personal area networks, and ubiquitous replacement for data cables [2][3]. UWB transmissions offer attractive advantages such as bypassing intermediate frequency stages (impulse-radio), and hence reducing complexity and cost of transceivers. Moreover, UWB systems are relieved of frequency coordination which allow them to operate within spectrum already occupied by existing radio services.

Due to the inherent nature of short duration pulses, UWB systems can resolve a large number of multipaths. Experimental evidence shows that the radio channel exhibits a clustering effect, where the arrival time of multipaths is modelled by two Poisson distributions. The first Poisson distribution gives the arrival for the first path of each cluster, and the second Poisson distribution gives the arrival of multipaths within each cluster. Therefore, based on the channel measurements, for the evaluation of different physical layer techniques of UWB systems, a modified Saleh-Valenzuela channel model for fading statistics has been proposed in [4].

Multipath propagation is considered as an advantage for UWB systems since the energy of different paths can be collected by a Rake receiver, hence, providing some diversity gain and protection against fading. As in any other wireless system, the detection of UWB signals requires channel estimation and synchronisation circuits. The synchronisation process has two steps of acquisition and tracking [5]. The acquisition is responsible for the coarse timing adjustment and the tracking is responsible to refine timing adjustment and keeping the synchronisation state.

A couple of estimation techniques for UWB systems has already been proposed in [6]. In this paper, we explore the issue of delay estimation and timing recovery for UWB systems using the maximum likelihood approach. Furthermore, two delay tracking circuits are presented. The first circuit is a non-coherent digital delay locked loop circuit and the second one is based on early-late technique.

The rest of the paper is organised as follows: Section II describes the UWB channel model. In Section III, the system model is illustrated and the principle of a UWB system is explained as the modulation/channel model. In Section IV, a closed form of the optimal ML estimation is derived. Section V describes the first and the second circuits. Section VI describes the performance of 
the proposed circuits based on the MSE. Finally, in Section VII conclusions are drawn.

\section{UWB ChANNEL MODEL}

The statistical channel model proposed by the IEEE 802.15 Working Group is employed. The path loss is assumed to be compensated in the link budget. Thus, a conventional wide sense stationary uncorrelated scattering (WSSUS) discrete channel model can be used. This discrete channel is the time variant impulse response, also known as the input delay-spread function or the first Bello function, assuming a system with finite delay resolution. Such a finite delay resolution is proportional to the inverse of the transmission channel bandwidth, where the received signal can be seen as a linear convolution of a transmitted signal with a band limited input-delay spread function given by

$$
h_{P_{\Delta \tau}}(t, \tau)=\sum_{n} h_{P_{\Delta \tau}}\left(t, \tau_{n}\right) \delta\left(t-\tau_{n}\right)
$$

Particularly for UWB channels, a clustering effect is reported in intensive measurement campaigns. Therefore, the channel response (1) is modelled as [5][7]

$$
h_{i}(t)=X_{i} \sum_{l=0}^{L} \sum_{k=0}^{K} \alpha_{k, l}^{i} \delta\left(t-T_{l}^{i}-\tau_{k, l}^{i}\right)
$$

where $h_{i}(t)$ is the $i^{\text {th }}$ channel realisation, $X_{i}$ is the log-normal shadowing effect, $\alpha_{k, l}$ is the multipath channel coefficient, and $T_{l}$ is the delay of the $l^{\text {th }}$ cluster. Moreover, $\tau_{k, l}$ is the delay of the $k^{\text {th }}$ multipath component relative to the $l^{\text {th }}$ cluster arrival time $T_{l}$ with respect to a reference at the receiver.

We assume perfect symbol synchronisation first. Hence, equation (2) can be simplified to

$$
h(t)=\sum_{l=1}^{L} \alpha_{l} \delta\left(t-\tau_{l}\right)
$$

which includes log-normal shadowing and multipath channel coefficients. The sparseness of the model can be mitigated by taking multipath signals that are $10 \mathrm{~dB}$ below the maximum peak.

\section{SySTEM MODEL}

UWB pulses experience multipath phenomena which can result in a degraded performance due to pulse distortion. UWB systems have a bandwidth exceeding $2 \mathrm{GHz}$ and can resolve multipath components with differential delays of a fraction of a nanosecond [8].

Two popular techniques for channelisation in UWB systems are time hopped spread spectrum (TH-SS) and direct sequence spread spectrum (DSSS) [9]. In TH-SS, a PN sequence is used to "pseudo-randomly" shift the position of a periodic pulse train from its nominal position using the low duty cycle of the pulse trains, whilst DS-SS UWB uses high duty cycles. For our purposes, the essential characteristics of both techniques are fundamentally similar; however, to illustrate the signal structure we consider a time hopping system which is most commonly described in the literature [10].

The baseband representation of a user's transmitted pulse is given by

$$
s(t)=\sum_{n=1}^{\infty} b_{n} w\left(t-n T_{s}\right)
$$

where the variable $b_{n} \in\{-1,1\}$ is the $n^{\text {th }}$ transmitted bit, $T_{s}$ is the symbol time, and $w(t)$ is the spreading waveform. The spreading waveform is composed of $M$ frames and is given by

$$
w(t)=\sum_{m=1}^{M} p\left(t-m T_{f}-c_{m} T_{c}\right)
$$

where $T_{f}$ is the frame time. Each frame is further divided into $N_{c}$ chips to give a chip interval of $T_{c}=$ $\frac{T_{f}}{N_{c}}$. The parameter $c_{m}$ is the spreading sequence and each user has a distinctive spreading sequence from a finite set $\left\{0,1, \ldots, N_{c-1}\right\}$. Furthermore, $p(t)$ characterises the shaping pulse, and a single pulse is transmitted in each frame time. We also assume that the channel consists of $L$ distinct and resolvable paths. In addition, we assume that the channel is a slow fading channel in which the multipath structure is not to change very frequently.

The received signal, $r(t)$, at the output of the channel is given by

$$
\begin{aligned}
r(t) & =s(t) * h(t)+n(t) \\
& =\sum_{n=1}^{\infty} \sum_{l=1}^{L} \alpha_{l} b_{n} w\left(t-n T_{s}-\tau_{l}\right)+n(t)
\end{aligned}
$$


where ${ }^{\prime} *$ indicates the convolution operation, and $n(t)$ is assumed to be additive white gaussian noise (AWGN) with zero mean and a two-sided power spectral density.

\section{MAXIMUM LiKeliHOOD APPROACH}

In this section timing recovery in UWB transmissions using the maximum likelihood technique is investigated. The essential feature of this approach is that the synchroniser's structure is the outcome of a mathematical optimisation problem, hence based on this mathematical optimisation problem, different structures can be proposed and designed.

The log-likelihood function of equation (6) with argument $\hat{\tau}$ (which can be regarded as a trial estimate of the parameter), is given by

$$
\begin{aligned}
& \wedge_{L}(\hat{\tau})=-\frac{1}{2 N_{0}} \int_{-\infty}^{\infty} r^{2}(t) d t+ \\
& \frac{1}{N_{0}}\left\{\sum_{n=1}^{\infty} \sum_{l=1}^{L} b_{n} \alpha_{l} \int_{-\infty}^{\infty} r(t) w\left(t-n T_{s}-\tau_{l}\right) d t\right\} \\
& -\left(\frac{1}{2 N_{0}} \sum_{n, n^{\prime}} \sum_{l, l^{\prime}} b_{n} b_{n^{\prime}} \alpha_{l} \alpha_{l^{\prime}}\right) . \\
& \int_{-\infty}^{\infty} w\left(t-n T_{s}-\tau_{l}\right) w\left(t-n^{\prime} T_{s}-\tau_{l^{\prime}}\right) d t
\end{aligned}
$$

Differentiating with respect to $\tau_{j}$, discarding irrelevant terms and equating the result to zero,

$$
\frac{\partial \wedge_{L}(\hat{\tau})}{\partial \tau_{j}}=0
$$

yields

$\sum_{n=1}^{\infty} b_{n} \alpha_{j} \frac{\partial}{\partial \tau_{j}} \int_{-\infty}^{\infty} r(t) w\left(t-n T_{\bar{s}}-\tau_{j}\right) d t-\sum_{n, n^{\prime}} \sum_{l^{\prime}} b_{n} b_{n^{\prime}} \alpha_{j} \alpha_{l^{\prime}}$

$$
\frac{\partial}{\partial \tau_{j}} \int_{-\infty}^{\infty} w\left(t-n T_{s}-\tau_{j}\right) w\left(t-n^{\prime} T_{s}-\tau_{l^{\prime}}\right) d t=0
$$

In order to solve this equation we can apply two different approaches:
A. First Approach: Derivative matched filter

Substituting $r(t)$ from equation (6) into equation (9) and rearranging yields

$$
\begin{aligned}
& \sum_{n, n^{\prime \prime}} \sum_{l^{\prime \prime}} b_{n} b_{n^{\prime \prime}} \alpha_{j} \frac{\partial}{\partial \tau_{j}} \int_{-\infty}^{\infty} w\left(t-n^{\prime \prime} T_{s}-\tau_{l^{\prime \prime}}\right) w\left(t-n T_{s}-\tau_{j}\right) d t \\
& +\sum_{n} b_{n} \alpha_{j} \frac{\partial}{\partial \tau_{j}} \int_{-\infty}^{\infty} n(t) w\left(t-n T_{s}-\tau_{j}\right) d t \\
& -\left(\sum_{n, n^{\prime}} \sum_{l^{\prime}} b_{n} b_{n^{\prime}} \alpha_{j} \alpha_{l^{\prime}}\right) \\
& \frac{\partial}{\partial \tau_{j}} \int_{-\infty}^{\infty} w\left(t-n T_{s}-\tau_{j}\right) w\left(t-n^{\prime} T_{s}-\tau_{l^{\prime}}\right) d t=0
\end{aligned}
$$

To proceed further, assume zero-mean and independent symbols so that

$$
E\left\{b_{n} b_{n+m}\right\}=\left\{\begin{array}{cc}
1 & m=0 \\
0 & m \neq 0
\end{array}\right.
$$

Now, taking the expectation of equation (10), the Timing Error Detector (TED) may be written as

$$
\arg \min _{\tau_{l}} \sum_{l} \int_{-\infty}^{\infty} w\left(t-n T_{s}-\tau_{l}\right) w^{\prime}\left(t-n T_{s}-\tau_{j}\right) d t
$$

where $w^{\prime}(t)$ is the derivative of $w(t)$. As an important result we observe that the delay estimation is accomplished when the output of the derivative matched filter is minimised with respect to the received signal.

\section{B. Second Approach: Early-late technique}

If the output of the derivative matched filter is written as

$$
\begin{aligned}
\dot{y}\left(n T_{s}+\tau_{j}\right) & =\frac{\partial y\left(n T_{s}+\tau_{j}\right)}{\partial \tau_{j}} \\
& =\frac{\partial}{\partial \tau_{j}} \int_{-\infty}^{\infty} r(t) w\left(t-n T_{s}-\tau_{j}\right) d t \\
& =\int_{-\infty}^{\infty} r(t) w^{\prime}\left(t-n T_{s}-\tau_{j}\right) d t
\end{aligned}
$$


and if the response of the the derivative matched filter with respect to $w(t)$ is written as

$$
\begin{aligned}
& \dot{R}_{j, l^{\prime}}^{n, n^{\prime}}=\frac{\partial R_{j, l^{\prime}}^{n, n^{\prime}}}{\partial \tau_{j}} \\
= & \frac{\partial}{\partial \tau_{j}} \int_{-\infty}^{\infty} w\left(t-n T_{s}-\tau_{j}\right) w\left(t-n^{\prime} T_{s}-\tau_{l^{\prime}}\right) d t \\
= & \int_{-\infty}^{\infty} w^{\prime}\left(t-n T_{s}-\tau_{j}\right) w\left(t-n^{\prime} T_{s}-\tau_{l^{\prime}}\right) d t
\end{aligned}
$$

then the derivatives of $y_{j}^{n}$ and $R_{j, l}^{n, n^{\prime}}$ in equations (13) and (14) can be approximated respectively as

$$
\begin{aligned}
& \dot{y}\left(n T_{s}+\tau_{j}\right)=\int_{-\infty}^{\infty} r(t) w\left(t-n T_{s}-\tau_{j}-\frac{\delta}{2}\right) d t \\
& -\int_{-\infty}^{\infty} r(t) w\left(t-n T_{s}-\tau_{j}+\frac{\delta}{2}\right) d t \\
= & y\left(n T_{s}+\tau_{j}+\frac{\delta}{2}\right)-y\left(n T_{s}+\tau_{j}-\frac{\delta}{2}\right) \\
= & \stackrel{+}{y}\left(n T_{s}+\tau_{j}\right)-\bar{y}\left(n T_{s}+\tau_{j}\right)
\end{aligned}
$$

and

$$
\begin{aligned}
\dot{R}_{j, l^{\prime}}^{n, n^{\prime}} & =\int_{-\infty}^{\infty} w\left(t-n T_{s}-\tau_{j}-\frac{\delta}{2}\right) w\left(t-n^{\prime} T_{s}-\tau_{l^{\prime}}\right) d t \\
& -\int_{-\infty}^{\infty} w\left(t-n T_{s}-\tau_{j}+\frac{\delta}{2}\right) w\left(t-n^{\prime} T_{s}-\tau_{l^{\prime}}\right) d t \\
& =R_{j, l^{\prime}}^{+}{ }^{n, n^{\prime}}-R_{j, l^{\prime}}{ }^{n, n^{\prime}}
\end{aligned}
$$

Substituting equations (15), (16) into equation (9) and taking the expectation of the expression yields the timing error detector, that is

$$
\stackrel{+}{R_{j, j}^{n, n}}=\bar{R}_{j, j}^{n, n}
$$

Hence, we have another formula for the delay estimation. The delay estimation is accomplished when the early and late components of the output of the matched filter become similar.

\section{TRACKING}

In this section, two non-coherent tracking loops are proposed to estimate the delays. The first approach is based on a non-coherent digital delay lock loop (DDLL). The received signal is matched filtered and the sampled output of the delayed and non-delayed components are used as a timing estimator.

In the second approach which is based on the early-late technique the received signal is correlated with a template signal with an arbitrary delay and the output of advanced/delayed offsets is compared to a threshold. Furthermore, based on the generated error signal the delay in the template signal is adjusted to estimate the received signal's delay. A false alarm occurs when a non-signal output exceeds the threshold.

\section{A. DDLL method}

The block diagram of this approach is shown in Fig. 1. The received signal is processed by passing it through a matched filter (MF). The output of the matched filter is sampled, then the output of the sampler is downsampled and the donwsampler's output is delayed by the amount of chip time. Further, the delayed and non-delayed outputs are PN code despread. To drive a proper loop error signal, the difference between the squared magnitudes of the delayed and non-delayed outputs are computed to provide a delay-offset estimate and to adjust sampling instants.

\section{B. Early-late method}

The block diagram of the second approach is shown in Fig. 2. The received signal is correlated with a locally generated pulse with an arbitrary time delay with respect to the received signal. The output of the correlators are integrated over the chip time and the early and late replica of the resulting signals are sampled. Ideally, if the timing error between the incoming signal and the locally generated pulse is zero, the energy measured at both branches should be identical due to the symmetry in the autocorrelation function. However, any timing offset leads to some difference in the measured energy at the early and late branches, and can be used to provide a delay-offset estimate and minimises this timing error. 


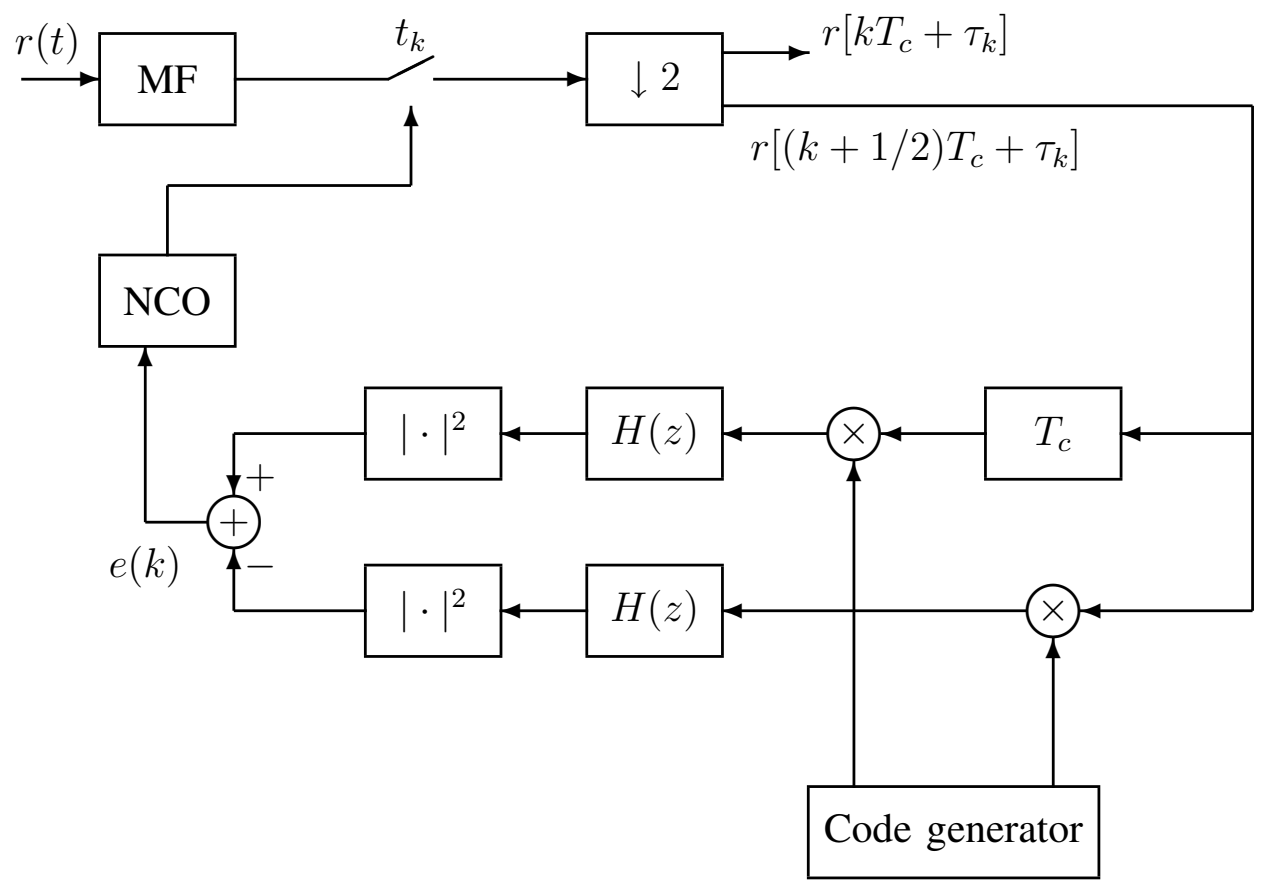

Fig. 1. Block diagram of the DDLL method.

\section{Vi. Simulation Results}

The performance of aforementioned circuits has been assessed by simulations and the following assumptions have been made.

- We used standard IEEE 802.15.3a channel models for the simulations, and 100 channel realisations have been considered.

- The gold training sequence is generated by the algorithm described in [11].

- We have chosen the monocycle pulse as the fifth derivative of a Gaussian function (if required, other pulse shapes can be used to improve the spectral properties); it can be expressed as

$$
p(t)=\left(t^{5}-10 t^{3}+15 t\right) e^{\frac{-t^{2}}{2 \sigma^{2}}}
$$

where $\sigma$ controls the pulse width and is chosen based on the FCC requirements.

- The channel impulse response is constrained to contain multipaths that are $10 \mathrm{~dB}$ below the maximum multipath peak.

- The pulse duration is $0.143 \mathrm{~ns}$.
- One pulse is transmitted every 100 ns, so intersymbol interference (ISI) is avoided.

Fig. 3 depicts the normalised mean square error (MSE) versus SNR for the first approach. The bits are transmitted as BPSK symbols using the mapping $0 \longrightarrow-1,1 \longrightarrow 1$.

Fig. 4 shows the normalised MSE versus SNR for the second approach.

The channel model CM1 represents a 0-4 m Line Of Sight (LOS) channel, CM2 a 0-4 m Non Line Of Sight (NLOS) channel, CM3 a 4-10 m NLOS channel and CM4 an extreme NLOS channel. As is evident, the CM1 results give a lower bound of the best performance for each scheme. Moreover, the performance of the first circuit is better than the second circuit but the complexity and its cost are higher than the second circuit.

\section{CONCLUSIONS}

We have investigated timing recovery techniques in ultra wideband systems based on the maximum likelihood approach. The results presented 


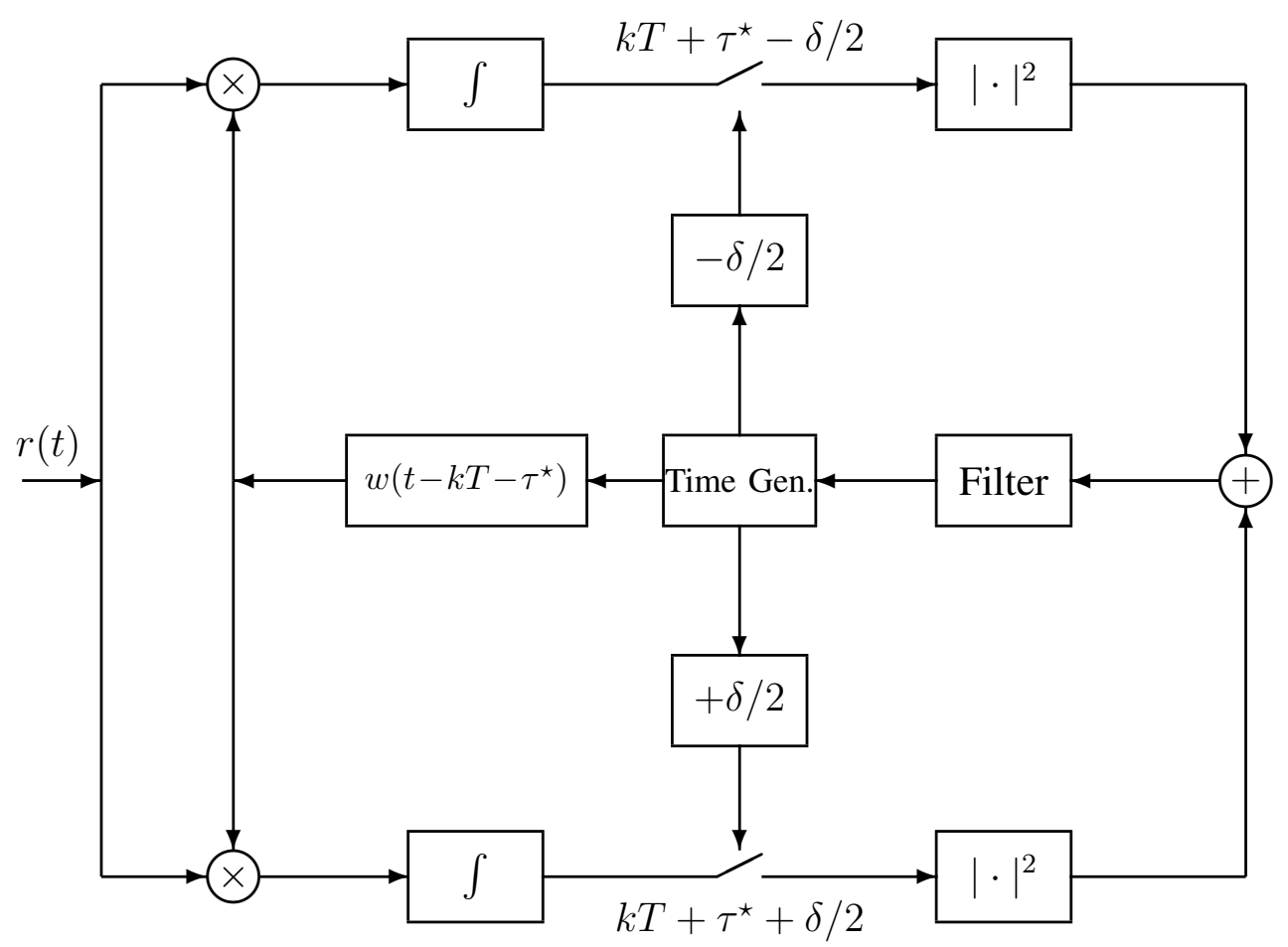

Fig. 2. Block diagram of the early-late method.

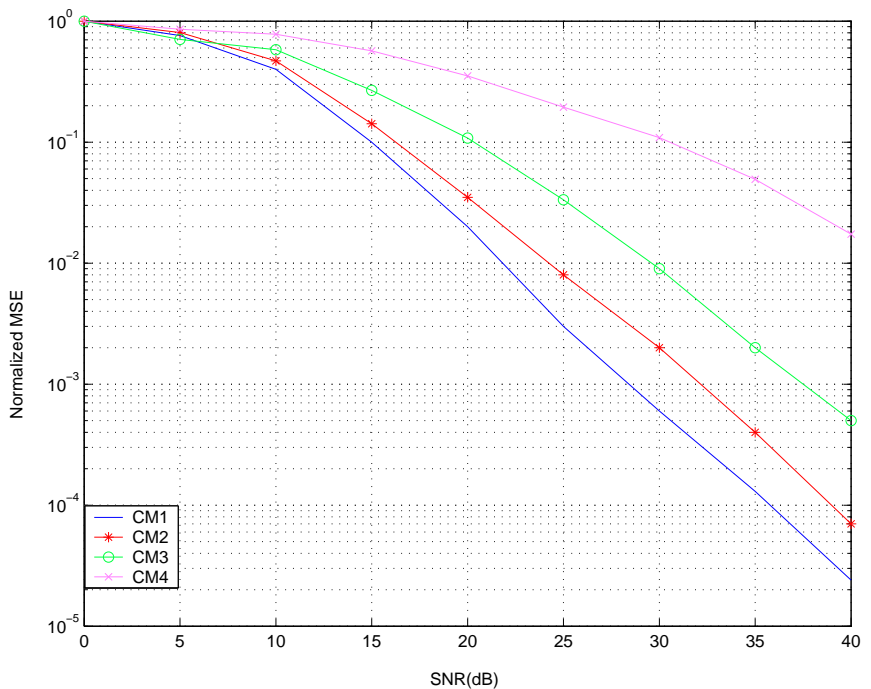

Fig. 3. Performance of the first circuit for CM1 to CM4

give an indication on the potential of different timing recovery schemes. The estimators in (12) and (17) enables timing synchronisation at desired and affordable complexity, that is both coarse and fine time estimation can be done (e.g., coarse time

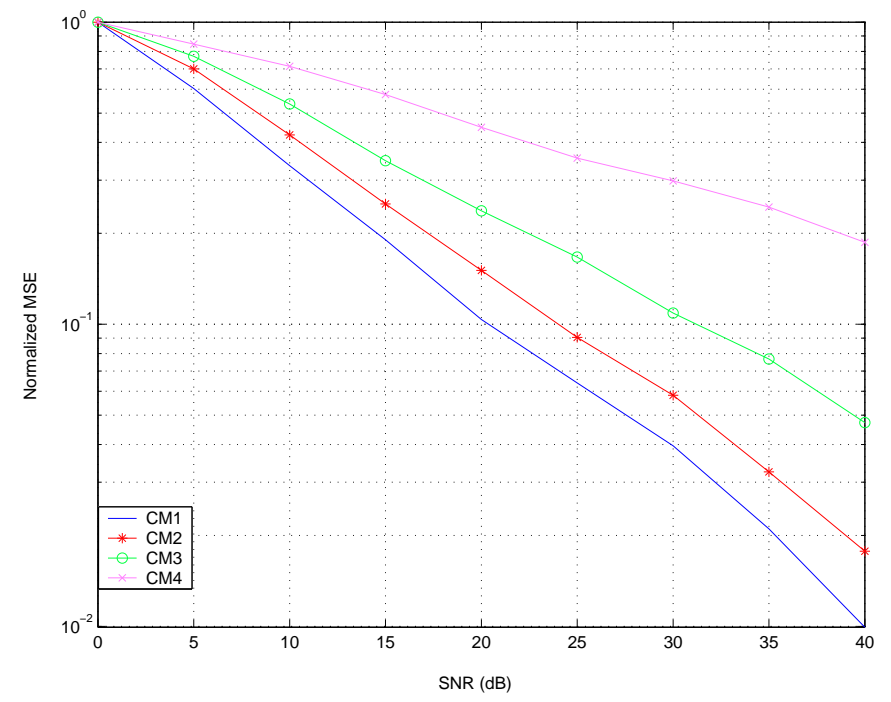

Fig. 4. Performance of the second circuit for CM1 to CM4

estimation can be done at the frame resolution and fine time estimation can be carried out at the chip resolution). Further, as non-coherent loops have been known to be more suitable for tracking with data modulation and channel imperfections 
than the coherent loops, two non-coherent synchronisers have been proposed. The performance of the proposed schemes and their complexity were illustrated with simulations. It should be noticed that the performance of tracking loop in Fig. 2 depends on the correlation function between the received signal and the template signal, hence careful design of this function improves the performance of circuit. Furthermore, the performance of both configurations depends on the type of loop filter used on the circuits.

Moreover, based on the estimators in (12) and (17) different circuit configurations depending on the application and the trade-off between performance and complexity can be proposed and designed.

\section{REFERENCES}

[1] FCC, First Report and Order 02-48, Feb. 2002.

[2] R. Dilmaghani, M. Ghavami, B. Allen, AH Aghvami, "Novel UWB pulse shaping using prolate spheroidal wave functions", 14th IEEE Proceedings on Personal Indoor Mobile Communications, Volume 1, Sept. 2003, Pages 602606.

[3] M. Ghavami, M. Lachlan and R. Kohno, Ultra Wideband Signals and Systems in Communication Engineering, John Wiley \& Sons, May 2004.

[4] J. Foerster, Editor, "Channel Modeling Subcommitee Report Final”, IEEE P802.15-02/490, February 2003, http://ieee802.org/15.

[5] M. K. Simon, M. Alouini, "Digital Communication over Fading Channels: A Unified Approach to Performance Analysis", Wiley-Interscience; 1st edition July 2000.

[6] R. Dilmaghani, M. Hernandez, M. Ghavami, R. Kohno, "Optimum Sequence Design and Performance Evaluation of Channel Estimation Techniques for Ultrawideband Systems", IEE Proc. Communications, Vol. 152, No. 2, April 2005, pp 241-246..

[7] D. Cassioli, M. Z. Win and A. F. Molisch, "A statistical model for the UWB indoor channel", in Proc. of IEEE Vehic. Tech. Conf., Rhodos, Greece, vol. 2, May 2001, pp.11591163.

[8] M. Win, R. Scholtz, "Ultra-Wide Bandwidth Time-Hopping Spread-Spectrum Impulse Radio for Wireless Multiple-Access communications,", IEEE Transactions on Communications, Vol. 48, April 2000, pp. 679-689.

[9] F. Ramirez-Mireles, "Performance of Ultra wideband SSMA Using Time-Hopping and M-ary PPM", IEEE Journal on Selected Areas in Communications, Vol. 19, June 2001 pp. 1186-1196.

[10] M. Z. Win and R. A. Scholtz, "Ultra-wide bandwidth timehopping spread-spectrum impulse radio for wireless multipleaccess communications," IEEE Trans. on Comm., vol. 48, April 2000, pp. 679-691.

[11] M. Hernandez, "Space-Time Multiuser Receivers for Wideband Code Division Multiple Access", Delft University Press, ISBN 904072301X, The Netherlands.

[12] R. L. Peterson, R. E. Ziemer, and D. E. Borth, Introduction to Spread Spectrum Communications. Englewood Cliffs, NJ: Prentice-Hall, 1995.

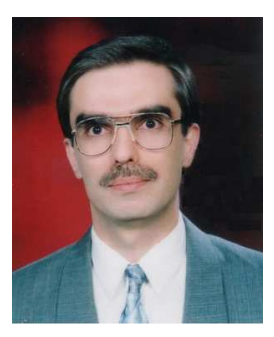

Mohammad Ghavami completed his Ph.D. degree with Great Distinction in electrical engineering at the University of Tehran, in 1993. During his Ph.D. studies he was awarded the DAAD scholarship at the University of Kaiserslautern, Germany from 1990 to 1993. From 1998 to 2000 he was a JSPS Postdoctoral fellow in Yokohama National University, Japan. From 2000 to 2002 he was a researcher at the Sony Computer Science Laboratories, Inc. in Tokyo, Japan. $\mathrm{He}$ is currently a Reader with the Ultra Wideband Communications Group, King's College, University of London.

$\mathrm{He}$ is the leading author of the book Ultra Wideband Signals and Systems and co-author of the book Adaptive Antenna Systems, and has published over 80 technical papers in major international journals and conferences on areas related to UWB systems, digital communications, adaptive filters and beam-space-time signal processing. He was the co-editor of a special issue of the IEE Proceedings on Communications on Ultra Wideband Systems Technologies and Applications, and associate editor of the special issue of IEICE Journal on ultra wideband communications systems. He is a senior member of IEEE and a member of IEE.

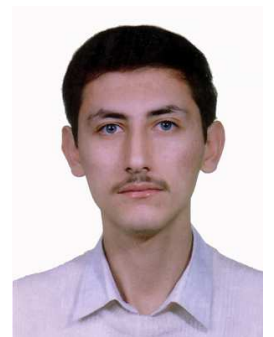

Reza S. Dilmaghani joined the Centre for Telecommunications Research (CTR), King's College London in January 2003 and received his in June 2006. Since May 2006, he has been a lecturer with the Ultra wideband Communications Group. He holds BSc and MSc degrees with distinction. Reza was a visiting researcher at the Japanese Government Lab, National Institute of Information and Communications Technology (NiCT), Japan during 2004 where he was researching on UWB channel modeling and channel estimation techniques for UWB systems. His interests include signal processing for communications, image processing, and wavelet theory. He has also published over 25 technical papers in recognised conferences and journals. 